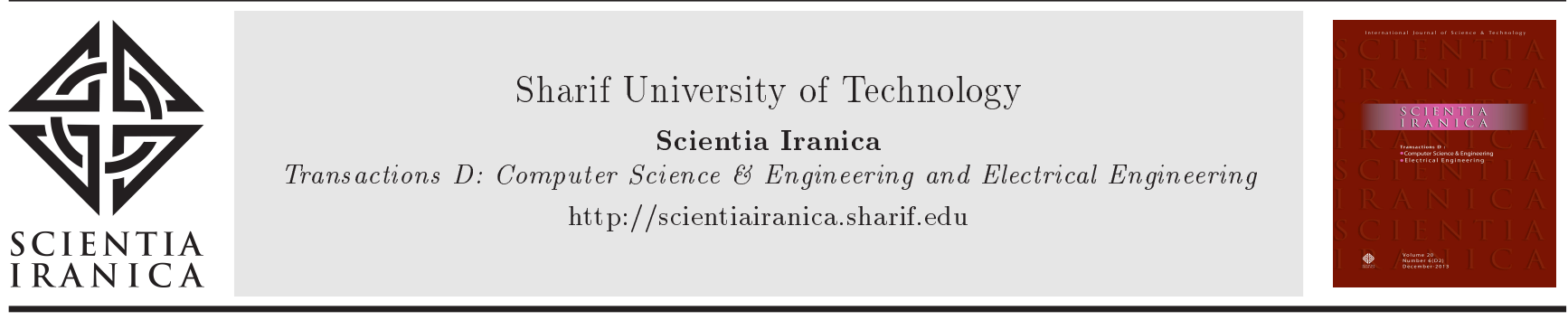

\title{
High-accuracy power sharing in parallel inverters in an islanded microgrid using modified sliding mode control approach
}

\author{
M.A. Bina, M. Gitizadeh*, and M. Mahmoudian \\ Faculty of Electrical Engineering, Shiraz University of Technology, Shiraz, Islamic Republic of Iran.
}

Received 2 June 2018; received in revised form 26 December 2018; accepted 16 February 2019

\author{
KEYWORDS \\ Distributed generation \\ unit; \\ Micro grid control; \\ Power sharing; \\ Sliding mode; \\ Control.
}

\begin{abstract}
The increased penetration level of Distributed Generation (DG) units in microgrids that feed large loads in parallel connections has led to developing the concept of power sharing. A microgrid's voltage and frequency in the islanded mode are controlled using an inverter with high inertia. Therefore, the internal control loop is executed in such a manner as to avoid overloading of all the DGs in the microgrid. Consequently, reactive power sharing error is eliminated and the voltage is also kept constant within the permissible range. This paper presents a modified control method based on the sliding mode approach. The proposed control method is tested using several disturbances and three scenarios. Also, the fractional order calculus is applied to the proposed control strategy to increase the convergence speed and system accuracy. Finally, the proposed method is compared to other well-known controlling approaches and the achieved results confirm its superiority.
\end{abstract}

(C) 2020 Sharif University of Technology. All rights reserved.

\section{Introduction}

Over the years, the penetration level of renewable and nonrenewable Distributed Generation (DG) units such as Wind Turbines (WTs), Fuel Cells (FCs), and photovoltaic arrays (PVs) has increased in power electricity networks. DG units play important roles in reducing pollutant gasses, attenuating transmitted power losses, and enhancing the power quality indices. All these benefits come to fore in large-scale networks. However, some of the main issues associated with power networks including line power flows and voltage deviation in the presence of DG units should be reviewed. Since

\footnotetext{
*. Corresponding author.

E-mail addresses: Mohammadaminbina@gmail.com (M.A.

Bina); gitizadeh@sutech.ac.ir (M. Gitizadeh);

m.mahmoudian@sutech.ac.ir (M. Mahmoudian)
}

doi: $10.24200 /$ sci.2019.51162.2038 a Micro-Grid (MG) is composed of various DG units, challenges of the high-penetration level of these units should be addressed [1].

Figure 1 represents the architecture of a typical MG with an Alternative Current (AC) system. PVs and energy storage systems are connected to $\mathrm{AC}$ network by DC-DC, AC-DC, and DC-AC converters with Local Controller (LC). Also, WTs are connected to Point of Common Coupling (PCC) by an AC-DC$\mathrm{AC}$ convertor. In the islanded operation mode, the local load should be supplied by renewable and nonrenewable DG units. In the grid-connected operation mode, the PCC is connected to the upstream highvoltage infinite bus. This bus has a major role in managing MG operation, while the PCC controls the power flow between the MG and the upstream network. Therefore, the protection management and the coordination insulation of PCC are highly necessary [2].

To guarantee the MG stability and its economic operation, the active and reactive powers of local loads 


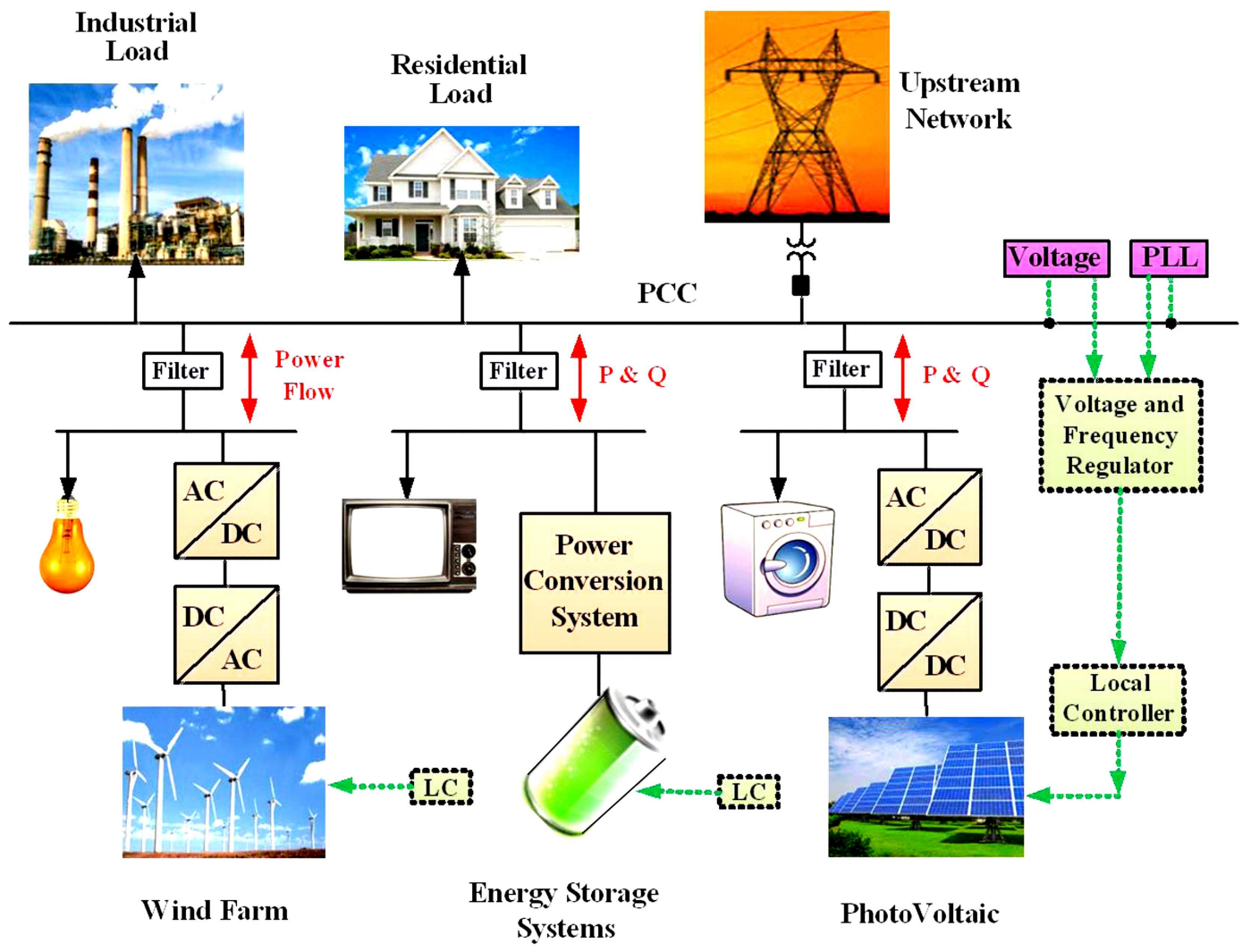

Figure 1. A general architecture of Micro-Grid (MG).

must be shared among DG units simultaneously and effectively. Droop controller is one of the renowned controllers in this context, which has been tested in different cases [3]. A modeling approach to explaining the normal and transient modes of network operation was provided in [4], called virtual synchronous generator, which is based on dynamic oscillation equations. However, the simplicity of the improved droop method for power sharing has made it a popular control algorithm among researchers in the islanded operation mode up to now.

In [4], a control methodology based on static compensators was provided to achieve more efficient power sharing among DG units. The steady state control of DG units was presented in [5]. To improve active and reactive power sharing based on separating and decoupling, in which a developed control would be needed, virtual impedance strategy was presented [6]. However, low-frequency dynamic problems of inverters like current-distortion dynamics, output voltage fluctuations, and attenuations were not investigated in papers $[5,6]$. In order to improve the dynamic stability of active and reactive power sharing, an optimized droop method was illustrated in [7]. Distributed droop method was introduced in [8] to adapt dynamic performance of power system characteristics to load variations. Furthermore, in order to obtain power sharing in complicated load sharing situations such as nonlinear load existence, a newer strategy based on the power regulation algorithm was introduced in [9]. In [10], power management through hierarchical control was introduced in the presence of DC-AC power inverters, for which the economic assessments were efficient.

In [11-13], voltage stability was investigated by considering significant noise and some control methods for P-V and Q-f properties based on droop controllers were presented. These approaches can be applied to grids with indefinite feeder impedance that extremely influences power sharing. When all DG units operate in the same frequency in a steady state, active power can be regulated properly by droop control method. Reactive power sharing in the presence of nonlinear loads and unbalanced feeder impedance is challenging [14]. In such circumstances, active power sharing might cause inappropriate reactive power flow among DG units. Due to the variation of active component of current drawn by load, the voltages of those buses connected to the loads vary, while reactive power is not shared. This may cause instability in power network [15]. To obtain successful reactive power sharing, methods that are based on droop control approaches are classified into three major categories: improved droop control methods [16], improved virtual impedance methods [17], and improved hierarchical control methods [18]. Table 1 compares the advantages 
Table 1. A comparison between power sharing control strategies.

\begin{tabular}{|c|c|c|}
\hline Control method & Advantages & Disadvantages \\
\hline $\begin{array}{l}\text { Droop control } \\
\text { method }\end{array}$ & $\begin{array}{l}\text { - High reliability } \\
\text { - Availability }\end{array}$ & $\begin{array}{l}\text { - Need a complex algorithm } \\
\text { - Inappropriate for complex loads } \\
\text { - Inappropriate for complex micro grids } \\
\text { - Communication line delay }\end{array}$ \\
\hline $\begin{array}{l}\text { Virtual impedance } \\
\text { method }\end{array}$ & $\begin{array}{l}\text { - Proper operation to reactive power sharing } \\
\text { - Acceptable for nonlinear and unbalanced loads }\end{array}$ & $\begin{array}{l}\text { - Difficult to calculate the coefficients } \\
\text { - Hard to design a suitable algorithm with high efficiency }\end{array}$ \\
\hline $\begin{array}{l}\text { Hierarchical control } \\
\text { method }\end{array}$ & $\begin{array}{l}\text { - Good recovery of voltage and frequency } \\
\text { - Power sharing with unknown feeders' impedance }\end{array}$ & $\begin{array}{l}\text { - Inappropriate operation for reactive power sharing } \\
\text { under unbalanced and nonlinear loads } \\
\text { - Need a complicated algorithm } \\
\text { - Communication line delay }\end{array}$ \\
\hline
\end{tabular}

and disadvantages of the aforementioned power sharing control methods.

Specifically, DG controlling methods in MGs can be customized into three layers. First layer: In the first layer, the initial control focuses on voltage and frequency stability. The controller is applied in this layer to power sharing without using telecommunication channels. Second layer: This layer removes voltage and frequency disturbances that may occur on waveforms in the previous step. Third layer: This layer manipulates the economic dispatch and electricity market problems such as optimal load flow and power generation plans between the microgrid and the upstream grid.

The structure of this paper is organized as follows: Section 2 presents the MG's problem formulation. Section 3 describes the grid under consideration and extracts state space equations. Section 4 illustrates the proposed control strategy and its stability considering Lyapunov function. Section 5 presents the fractionalorder back-stepping sliding mode controller and the last section analyzes and investigates the simulation results. Consequently, a good comparison between a number of well-known control methods is expressed. Finally, the relevant conclusion is presented.

\section{Fractional order definitions}

Fractional-order calculations play an important role in various scientific fields. The applications of these calculations in control and electrical engineering are too many. Recently, scientists have shown that fractionalorder equations can model various phenomena more appropriately than integer order calculations. In reality, fractional-order controllers are powerful instruments to control electrical systems with complex dynamic conditions. The calculations for derivatives and integrals have some different definitions in fractional-order calculations. The most depleted and the most important fractional-order calculation methods are GrunwaldLetnikov, Riemann-Liouville, and Caputo [19]. In this paper, to design a fractional-order sliding mode controller, the derivative and integral of the fractional order with Caputo definition will be used [19].

Definition 1. Fraction derivative of Caputo is formulated as Eq. (1), in which $m$ is a positive number [19]:

$$
{ }_{0}^{c} D_{t}^{\alpha} x(t)=\frac{1}{\Gamma(m-\alpha)} \int_{0}^{t}(t-\tau)^{m-\alpha-1} x^{(m)}(\tau) d \tau,
$$

where $m-1<\alpha<m$. ${ }_{0}^{c} D_{t}^{\alpha} x(t)$ shows derivative of order $m$ of function $x(t)$ with Caputo definition from 0 to $t[19]$.

Definition 2. Gamma function is expressed as Eq. (2):

$$
\Gamma(z)=\int_{0}^{\infty} e^{-t} t^{z-1} d t .
$$

Definition 3. Fractional integral is expressed as Eq. (3):

$$
D_{0, t}^{-\alpha} x(t)=\frac{1}{\Gamma(\alpha)} \int_{0}^{t}(t-\tau)^{\alpha-1} x(\tau) d \tau
$$

Definition 4. Some important properties of Caputo definition are used later in the text [20]:

$$
\begin{aligned}
& { }_{0}^{c} D_{t}^{\alpha} D_{0, t}^{-\alpha} x(t)=x(t) ; \quad m=1, \\
& D_{0, t 0}^{-\alpha c} D_{t}^{\alpha}=x(t)-\sum_{k=0}^{m-1}, \\
& \frac{t^{k}}{k !} x^{(x)}(0),{ }_{0}^{c} D_{t 0}^{\alpha c} D_{t}^{n} x(t)={ }_{0}^{c} D_{t}^{\alpha+n} x(t) ; \quad n \in N, \\
& L\left\{{ }_{0}^{c} D_{t}^{\alpha} x(t)\right\}=s^{\alpha} X(s)-\sum_{k=0}^{m-1} s^{(\alpha-k-1)} x^{(k)}(0) .
\end{aligned}
$$

\section{Definition of case study: A typical microgrid}

Figure 2 depicts the schematic diagram of a typical MG and its control scheme. As illustrated earlier, the exchange of active and reactive powers with the MG has been compared to their reference values. Then, the 


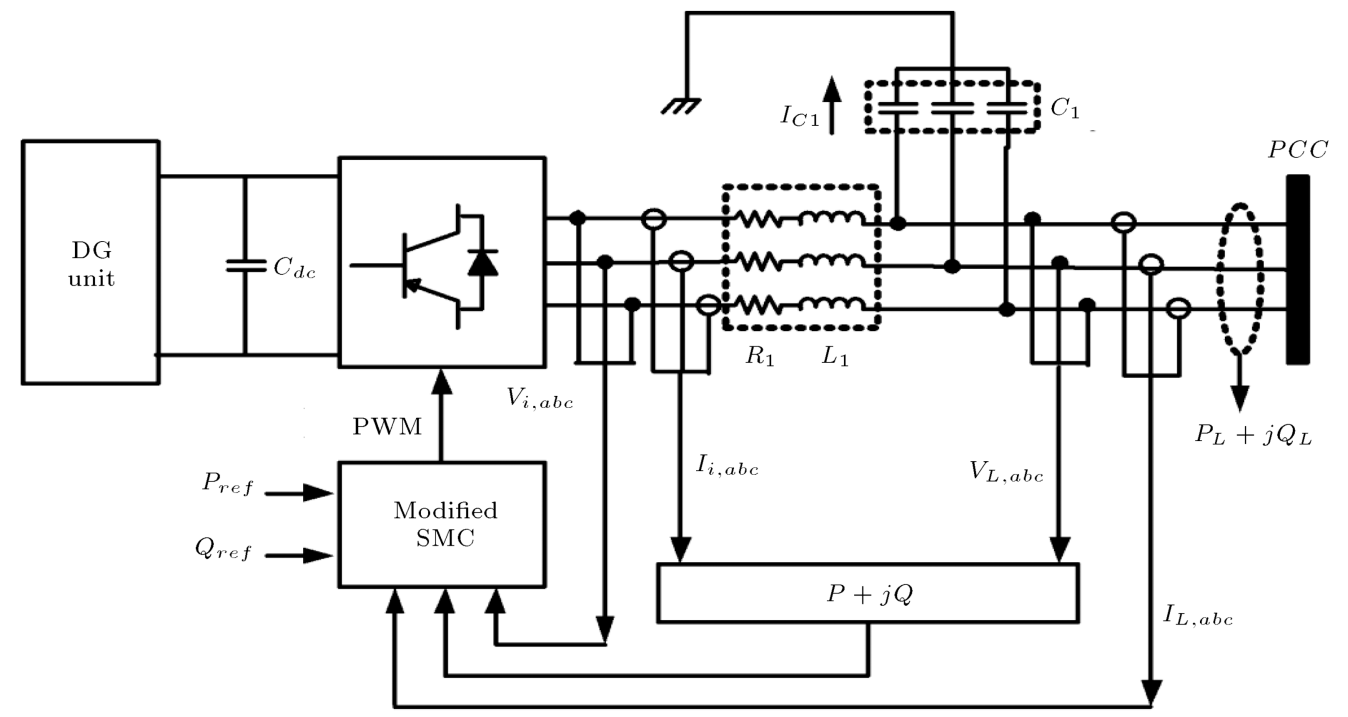

Figure 2. Control block diagram of Micro-Grid (MG).

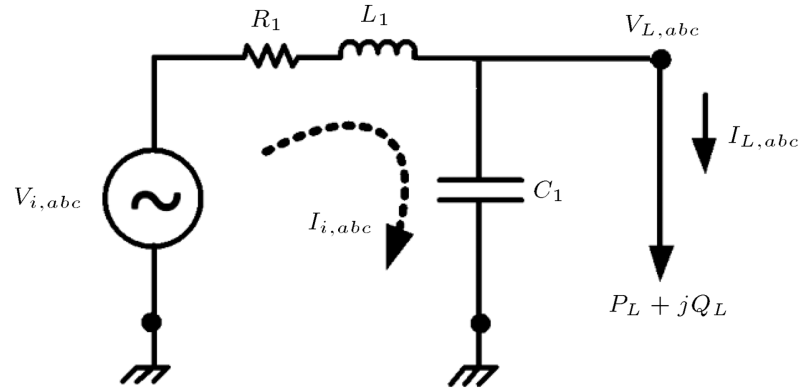

Figure 3. Equivalent circuit of Micro-Grid (MG).

proposed controller generates a firing angle of inverter switches. Power balance between DGs in MG and loads will keep the DC link voltage constant. By considering equivalent circuit of $\mathrm{MG}$ shown in Figure 3, the following formula can be achieved:

$$
\begin{aligned}
& \frac{d I_{i, a b c}}{d t}=-\frac{R_{1}}{L_{1}} I_{i, a b c}+\frac{1}{L_{1}}\left(V_{i, a b c}-V_{L, a b c}\right), \\
& \frac{d V_{L, a b c}}{d t}=\frac{1}{C_{1}}\left(I_{i, a b c}-I_{L, a b c}\right),
\end{aligned}
$$

where $V_{i, a b c}$ is the output voltage of the inverter across the filter, $V_{L, a b c}$ is the capacitor voltage or PCC voltage, $I_{i, a b c}$ is the current flow through the RL filter, and $I_{L, a b c}$ is the three-phase load current. Thus, the active and reactive powers exchanged with the $\mathrm{MG}$ can be calculated as follows:

$$
\begin{aligned}
P_{M G}= & P=V_{L a} I_{i a}+V_{L b} I_{i b}+V_{L c} I_{i c}, \\
Q_{M G}= & Q=\frac{V_{L a}\left(I_{i b}-I_{i c}\right)}{\sqrt{3}}+\frac{V_{L b}\left(I_{i c}-I_{i a}\right)}{\sqrt{3}} \\
& +\frac{V_{L c}\left(I_{i a}-I_{i b}\right)}{\sqrt{3}}
\end{aligned}
$$

where:

$$
\begin{aligned}
& P_{M G}=P_{D G 1}+P_{D G 2}+P_{D G 3}+P_{D G 4}, \\
& Q_{M G}=Q_{D G 1}+Q_{D G 2}+Q_{D G 3}+Q_{D G 4} .
\end{aligned}
$$

Hiring park transformation matrix is obtained as follows:

$$
\begin{aligned}
& \frac{d I_{i, d}}{d t}=-\frac{R_{1}}{L_{1}} I_{i, d}+\frac{1}{L_{1}}\left(V_{i, d}-V_{L, d}\right)+\omega I_{i, q} \\
& \frac{d I_{i, q}}{d t}=-\frac{R_{1}}{L_{1}} I_{i, q}+\frac{1}{L_{1}}\left(V_{i, q}-V_{L, q}\right)-\omega I_{i, d} .
\end{aligned}
$$

Therefore, to achieve control parameters like modulation index $\left(m_{a}\right)$ and its phase angle $(\beta)$, we can define $V_{i, d}=m_{a} V_{d c} \cos (\beta)$ and $V_{i, q}=m_{a} V_{d c} \sin (\beta)$. Therefore, Eq. (6) will convert to:

$$
\begin{aligned}
& P=P_{D G 1}+P_{D G 2}+P_{D G 3}+P_{D G 4}=V_{i, d} I_{i, d}+V_{i, q} I_{i, q}, \\
& Q=Q_{D G 1}+Q_{D G 2}+Q_{D G 3}+Q_{D G 4}=V_{i, q} I_{i, d}-V_{i, q} I_{i, d} .
\end{aligned}
$$

Finally, the dynamic performance of this MG is formulated as follows:

$$
\begin{aligned}
\frac{d P}{d t}= & -\frac{R_{1}}{L_{1}} P-\omega Q+\frac{1}{L_{1}}\left(V_{i, d} V_{L, q}-V_{i, q} V_{L, d}\right), \\
\frac{d Q}{d t}= & -\frac{R_{1}}{L_{1}} Q+\omega P+\frac{1}{L_{1}}\left(V_{i, d} V_{L, d}+V_{i, q} V_{L, q}\right. \\
& \left.-V_{L, d}^{2}-V_{L, q}^{2}\right), \\
\frac{d V_{L, d}}{d t}= & \frac{1}{C_{1}}\left(I_{i, d}-I_{L, d}\right) .
\end{aligned}
$$

Here, the state space dynamic modeling with three decision variables $\left(P, Q, V_{L}\right)$ is finalized. By substituting $x_{1}=P, x_{2}=Q$, and $x_{3}=V_{L}$ and defining $x_{1 d}, x_{2 d}$, and $x_{3 d}$ as the desired vectors, it can be concluded that: 


$$
\begin{aligned}
& z_{1}=x_{1}-x_{d}, \\
& z_{2}=x_{2}-x_{2 d}, \\
& z_{3}=x_{3}-x_{3 d} .
\end{aligned}
$$

The $Z$ vector contains the solutions which should be minimized by the proposed control process.

\section{Fractional order back-stepping sliding mode controller}

Sliding Mode Control (SMC) is widely used in nonlinear systems to track the reference signal accurately. SMC has many benefits including its good performance in the presence of external and internal noises. Backstepping algorithm intrinsically has the ability to deal with inconsistent confusion and can be combined with the sliding mode controller in order to increase the robust performance. Many studies have been conducted recently on fractional-order controllers [21]. One of the advantages of a fractional-order controller is the proper freedom degree of decision parameters. Based on backstepping algorithm theory [22], tracking the reference signal for minimizing the error signal in each system has a pseudo-control rule. The error signal is defined as (12) and then, back-stepping algorithm is applied to the Maglo approach as follows:

$$
\xi=z_{1}=x_{1}-x_{1 d} .
$$

Pseudo-control rule $\phi_{1}$ for mode $z_{1}$ considering MG dynamic model is determined in Eq. (13):

$$
\phi_{1}=-\lambda_{1} \xi_{1},
$$

where $\lambda_{1}$ is a constant and positive coefficient. Here, the dynamic equation of network power disturbance is determined in Eq. (14):

$$
\xi_{2}=z_{2}-\phi_{1} \text {. }
$$

The positive defined function written in Eq. (15) illustrates the stability of state $z_{1}$ under pseudo-control rule $\phi_{1}$ as follows:

$$
V_{1}=\frac{1}{2} \xi_{1}^{2}
$$

By deriving Eq. (15) and substituting it in Eqs. (12) and (16) will be achieved as follows:

$$
\dot{V}_{1}=\xi_{1} \dot{\xi}_{1}=\xi_{1} z_{2} \text {. }
$$

Also, by substation of Eqs. (13) and (14) in Eq. (16), we will have:

$$
\dot{V}_{1}=\xi_{1}\left(\xi_{2}+\phi_{1}\right)=\xi_{1} \xi_{2}-\lambda_{1} \xi_{1}^{2} .
$$

Derivative of the positive definite function $\left(\dot{V}_{1}\right)$ is negative and semi-definite; therefore, state $z_{2}$ is stable under pseudo-control rule according to Eq. (13). This process is repeated for modes $z_{2}$ and $z_{3}$, similarly. Pseudo-control rule for mode $z_{3}$ is determined in Eq. (18):

$$
\phi_{2}=\phi_{1}-\lambda_{2} \xi_{2}-\xi_{1}
$$

According to the back-stepping algorithm, dynamic disturbance of $\xi_{3}$ is defined as follows:

$$
\xi_{3}=z_{3}-\phi_{2} \text {. }
$$

By specifying the positive definite function of $V_{2}$, evaluating the stability of $z_{2}$ in system dynamic equations mentioned in Eq. (11) is investigated below according to the pseudo-control rule:

$$
V_{2}=V_{1}+\frac{1}{2} \xi_{2}^{2}
$$

By differentiating Eq. (20) and inserting Eqs. (17)-(19) into Eq. (20), we will have:

$$
\begin{aligned}
\dot{V}_{2} & =\dot{V}_{1}+\xi_{2} \dot{\xi} 2=\left(\xi_{1} \xi_{2}-\lambda_{1} \xi_{1}^{2}\right)+\xi_{2}\left(\dot{z}_{1}-\dot{\phi}_{1}\right) \\
& =\left(\xi_{1} \xi_{2}-\lambda_{1} \xi_{1}^{2}\right)+\xi_{2}\left(\xi_{3}+\phi_{2}-\dot{\phi}_{1}\right) \\
& =\left(\xi_{1} \xi_{2}-\lambda_{1} \xi_{1}^{2}\right)+\xi_{2}\left(\xi_{3}-\lambda_{2} \xi_{2}-\xi_{1}\right) \\
& =\xi_{2} \xi_{3}-\lambda_{1} \xi_{1}^{2}-\lambda_{2} \xi_{2}^{2} .
\end{aligned}
$$

Differentiating positive certain function $\left(\dot{V}_{2}\right)$ results in a negative semi-definite function. Therefore, state $z_{2}$ is stable under pseudo-control rule. In order to implement the SMC theory for calculating equivalent control rule, a proper sliding surface should be chosen. In this regard, to calculate the equivalent control rule properly, which ensures the stability of the control system, a sliding surface of fractional order is proposed as Eq. (22):

$$
\sigma=k_{1}^{c} D_{t}^{-\alpha} \xi_{3}+\xi_{3} .
$$

In order to calculate the equivalent control rule, Eq. (19) is substituted in derivative of Eq. (22); thus, we have:

$$
\begin{aligned}
& \dot{\sigma}=k_{1}^{c} D_{t}^{1-\alpha} \xi_{3}+\dot{\xi}_{3}, \\
& \dot{\sigma}=k_{1}^{c} D_{t}^{1-\alpha} \xi_{3}+\dot{z}_{3}-\dot{\phi}_{2} .
\end{aligned}
$$

Dynamic equations of the control system that are illustrated in Eq. (11) are substituted in Eq. (23); therefore, the system input $u$ is found as in Eq. (23). The SMC theory is employed to keep the system modes on the sliding surface or close to it.

This condition is achieved when $\sigma=\dot{\sigma}=0$. By applying these conditions to Eq. (24), the equivalent control rule is changed as follows: 


$$
\begin{aligned}
& \dot{\sigma}=k_{1}^{c} D_{t}^{1-\alpha} \xi_{3}+f(z)+g(z) u-\dot{\phi}_{2}=0, \\
& u_{e q}=-(g(z))^{-1}\left(k_{1}^{c} D_{t}^{1-\alpha} \xi_{3}+f(z)-\dot{\phi}_{2}\right) .
\end{aligned}
$$

By applying Eq. (20) to the system, the state modes converge to the sliding surface, which are determined by fractional order and remain on this surface. However, if any turbulence is applied to the system, or a parametric indeterminate occurs, there is no guarantee that system modes would converge to the determined sliding surface again. To resolve this drawback, the switching control rule is defined as Eq. (27):

$$
u_{s w}=-(g(z))^{-1}\left(k_{1} \sigma+k_{2} \operatorname{sign}(\sigma)\right) \text {. }
$$

Therefore, in order to stabilize and create proper tracking with robust performance in the system, control rule obtained by the summation of Eqs. (26) and (27) is applied to inverter switches. Then, we have:

$$
\begin{aligned}
u=- & (g(z))^{-1}\left(k_{1}^{c} D_{t}^{-\alpha} \xi_{3}+f(z)-\dot{\phi}_{2}+k_{1} \sigma\right. \\
& \left.+k_{2} \operatorname{sign}(\sigma)\right) .
\end{aligned}
$$

SMC theory has an unfavorable phenomenon in control rule called chattering, which causes discontinuity in the control rule. Chattering occurs due to discontinuous property of function sign in switching control rule [23]. To resolve this deficiency in control rule presented in Eq. (28), instead of sign function, a continuous function like saturation function $(\operatorname{sat}(\sigma / \epsilon))$ could be substituted.

Theory 1. Microgrid system definition illustrated in Eqs. (10) and (11) is stable by applying fractional-order back-stepping sliding mode controller and control rule Eq. (28). In other words, system modes will converge to fractional-order sliding surface described in Eq. (22).

Proof: According to the SMC theory, the proposed controller stability is evaluated using Lyapunov function. To this end, consider positive defined function as presented in Eq. (29):

$$
V_{3}=V_{2}+\frac{1}{2} \sigma^{2} \text {. }
$$

Derivative of Eq. (29) is calculated as Eq. (30):

$$
\dot{V}_{3}=\dot{V}_{2}+\sigma \dot{\sigma} \text {. }
$$

Inserting Eqs. (24) and (21) in Eq. (30) will lead to:

$$
\begin{aligned}
\dot{V}_{3}= & \xi_{2} \xi_{3}-\lambda_{1} \xi_{1}^{2}-\lambda_{2} \xi_{2}^{2}+\sigma\left(k_{10}^{c} D_{t}^{1-\alpha} \xi_{3}+f(z)\right. \\
& \left.+g(z) u-\dot{\phi}_{2}\right) .
\end{aligned}
$$

In order to calculate the best control rule, Eq. (28) should be substituted in Eq. (31):

$$
\begin{aligned}
\dot{V}_{3}= & \xi_{2} \xi_{3}-\lambda_{1} \xi_{1}^{2}-\lambda_{2} \xi_{2}^{2}+\sigma\left\{k_{1}^{c} D_{t}^{1-\alpha} \xi_{3}+f(z)\right. \\
& -\dot{\phi}_{2}+g(z)\left[-(g(z))^{-1}\left(k_{10}^{c} D_{t}^{-\alpha} \xi_{3}+f(z)\right.\right. \\
& \left.\left.\left.-\dot{\phi}_{2}+k_{1} \sigma++k_{2} \operatorname{sign}(\sigma)\right)\right]\right\} .
\end{aligned}
$$

That will be simplified as follows:

$$
\dot{V}_{3}=\xi_{2} \xi_{3}-\lambda_{1} \xi_{1}^{2}-\lambda_{2} \xi_{2}^{2}-k_{1} \sigma^{2}-k_{2}|\sigma| \text {. }
$$

According to Lyapunov's stability theory, to confirm the stability of a system, it is necessary that a certain positive function derivative must be definite negative. Since all the terms in Eq. (33) have a definite mathematical sign and $\xi_{2} \xi_{3}$ is not determined with a certain sign, considering certain positive matrix laws, it is proved that Eq. (33) will be certain negative and the system is asymptotically stable. After implementing the proposed sliding surface explained in Eqs. (22)(33), we obtain the following:

$$
\begin{aligned}
\dot{V}_{3}= & \xi_{2} \xi_{3}-\lambda_{1} \xi_{1}^{2}-\lambda_{2} \xi_{2}^{2}-k_{1}\left(k_{1}^{c} D_{t}^{-\alpha} \xi_{3}+\xi_{3}\right)^{2} \\
& -k_{2}|\sigma|+\xi_{2} \xi_{3}-\lambda_{1} \xi_{1}^{2}-\lambda_{2} \xi_{2}^{2}-k_{1} \xi_{3}^{2} \\
& -k_{1}\left(k_{1}^{c} D_{t}^{-\alpha} \xi_{3}\right)^{2}-k_{2}|\sigma|-2 k_{10}^{2 c} D_{t}^{-\alpha} \xi_{3} \\
& =-E^{T} Q E-k_{1}\left(k_{1}{ }_{0}^{c} D_{t}^{-\alpha} \xi_{3}\right)^{2}-2 k_{10}^{2 c} D_{t}^{-\alpha} \xi_{3} \\
& -k_{2}|\sigma|,
\end{aligned}
$$

where $E=\left[\begin{array}{lll}\xi_{1} & \xi_{2} & \xi_{3}\end{array}\right]$.

To have a certain negative Lyapunov function of derivative from Eq. (34), the matrix $Q$ must be certain positive. In the following, it is shown that matrix $Q$ is certain positive with some criteria. Matrix $Q$ is determined as follows:

$$
Q=\left[\begin{array}{ccc}
\lambda_{1} & 0 & 0 \\
0 & \lambda_{2} & -0.5 \\
0 & -0.5 & k_{1}
\end{array}\right]
$$

To have a certain positive matrix $Q$, all the leading principal minors of $Q$ need be positive. Thus, all leading principal minors of matrix $Q$ are defined as follows:

$$
\begin{aligned}
& \lambda_{1}>0 ;\left|\begin{array}{cc}
\lambda_{1} & 0 \\
0 & \lambda_{2}
\end{array}\right|=\lambda_{1} \lambda_{2}>0 \\
& Q=\lambda_{1}\left|\begin{array}{cc}
\lambda_{2} & -0.5 \\
-0.5 & k_{1}
\end{array}\right|=\lambda_{1}\left(\lambda_{2} k_{1}-0.25\right)>0 .
\end{aligned}
$$

Finally, if the coefficients $\lambda_{1}, \lambda_{2}$, and $k_{1}$ are applied to Eq. (36), then matrix $Q$ is certain positive 
and term $-E^{T} Q E$ will be certain negative in Lyapunov function derivative. According to [24], it was found that $-2 K_{1} k_{1} \xi_{30}^{c} D_{t}^{-\alpha} \xi_{3}<0$ and $k_{1}$ was determined more than zero. Therefore, according to Lyapunov's stability theory, this system will be stable by using the proposed controller. Theory 1 shows that our system modes converge to the fractional-order sliding surface of Eq. (22). In Theory 2, the process of converging the fractional-order sliding modes to zero will be investigated. To study the stability of the proposed sliding mode as described in Eq. (22), a new generalized Lyapunov stability theory and presented methods in [25] are used.

Theory 2. Fractional-order back-stepping sliding mode represented by Eq. (22) is stable and it converges to $\sigma=0$.

Proof: Assume that the fractional-order sliding mode is as the following:

$$
\sigma(t)=k_{10}^{c} D_{t}^{-\alpha} \xi_{3}+\xi_{3}=0 .
$$

Based on the definitions presented in Eq. (4), after differentiating the fractional order of Eq. (37) and using the new generalized Lyapunov theory for fractional order, it was proved that the fractional order sliding mode converged to zero as follows:

$$
k_{1}^{c} D_{t 0}^{\alpha c} D_{t}^{-\alpha} \xi_{3}+{ }_{0}^{c} D_{t}^{\alpha} \xi_{3}=0 ; \quad{ }_{0}^{c} D_{t}^{\alpha} \xi_{3}=-k_{1} \xi_{3} .
$$

Definite function at Eq. (39) is utilized to prove the stability of error $\xi_{3}$ in fractional-order dynamic equations. Therefore, we have:

$$
V=\frac{1}{2} \xi_{3}^{2} .
$$

From Eq. (39), the fractional-order derivative ${ }_{0}^{c} D_{t}^{\alpha}$ is taken and substituted in Eq. (38); therefore, we have:

$$
{ }_{0}^{c} D_{t}^{\alpha} V=\frac{1}{2}{ }_{0}^{c} D_{t}^{\alpha} \xi_{3}^{2}<\xi_{30}^{c} D_{t}^{\alpha} \xi_{3}=-k_{1} \xi_{3}^{2}<0 .
$$

According to the proposed definitions for the new generalized Lyapunov stability as well as the fractional-order derivative of this Lyapunov function, it is confirmed that ${ }_{0}^{c} D_{t}^{\alpha} V$ is smaller than the negative term $\left(-k_{1} \xi_{3}^{2}\right)$. As a result, the fractional-order sliding mode of Eq. (22) has a limited domain and converges to zero asymptotically.

\section{Simulation results}

A single-line diagram of a typical MG containing four DG units is shown in Figure 4. These DG units are connected to four different feeders to supply different load types. The main characteristics of DG units are presented in Tables 2 and 3 .

The specifications of loads and impedances of lines

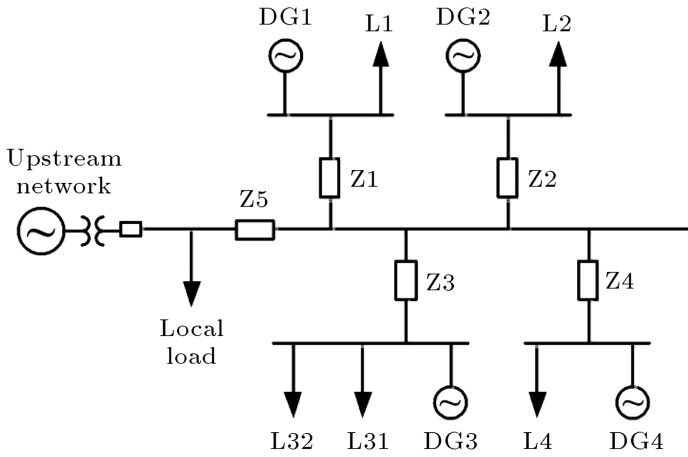

Figure 4. Microgrid scheme under consideration.

Table 2. Specifications of the tested Micro-Grid (MG).

\begin{tabular}{cc}
\hline Micro-grid properties & Parameters \\
\hline DC voltage & $500(\mathrm{~V})$ \\
MG voltage & $380(\mathrm{~V})$ (line to line) \\
& $220(\mathrm{~V})($ phase to ground) \\
Frequency & $50(\mathrm{~Hz})$ \\
\hline
\end{tabular}

Table 3. Distributed Generation (DG) characteristics and capacities.

\begin{tabular}{cc}
\hline DG index & Capacity (kVA) \\
\hline DG $\mathbf{1}$ & $40+j 20$ \\
DG $\mathbf{2}$ & $50+j 25$ \\
DG $\mathbf{3}$ & $60+j 30$ \\
DG 4 & $70+j 35$ \\
\hline
\end{tabular}

Table 4. Load characteristics.

\begin{tabular}{cc}
\hline Load index & Power (kVA) \\
\hline Load $\mathbf{1}$ & $10+j 5$ \\
Load 2 & $15+j 7.5$ \\
Load $\mathbf{3}$ & $L 31=6+j 3$ \\
& $L 32=14+j 7$ \\
Load 4 & $25+j 12.5$ \\
Local Load & $91+j 45.5$ \\
\hline
\end{tabular}

are given in Tables 4 and 5, respectively. In order to validate the proposed control scheme for power sharing between DG units, three different case studies are investigated. First, the achieved results are compared against those obtained by basic droop control methods. In the second case study, load variation effect on the presented control strategy is evaluated. Finally, a DG unit power outage will be investigated and power sharing is presented in the third case study. Table 6 represents the required data for the proposed control scheme.

\subsection{Case study 1}

In this case study, it is assumed that the MG is 
Table 5. Impedance of lines.

\begin{tabular}{cc}
\hline Line impedance & Per unit \\
\hline Impedance of Line $\mathbf{1}(\mathbf{Z 1})$ & $0.0087+j 0.039$ \\
Impedance of Line $\mathbf{2}(\mathrm{Z2})$ & $0.0298+j 0.098$ \\
Impedance of Line $\mathbf{3}(\mathrm{Z3})$ & $0.0301+j 0.124$ \\
Impedance of Line $\mathbf{4}(\mathrm{Z4})$ & $0.0385+j 0.266$ \\
Impedance of Line $\mathbf{5}(\mathbf{Z 5})$ & $0.0421+j 0.295$ \\
\hline
\end{tabular}

Table 6. Control strategy parameters.

\begin{tabular}{cc}
\hline Parameters & Value \\
\hline $\boldsymbol{\lambda}_{\mathbf{1}}$ & 24 \\
$\boldsymbol{\lambda}_{\mathbf{2}}$ & 21 \\
$\boldsymbol{\gamma}_{\mathbf{1}}$ & 52 \\
$\boldsymbol{\gamma}_{\mathbf{2}}$ & 82 \\
$\boldsymbol{k}_{\mathbf{1}}$ & 0.12 \\
$\boldsymbol{\alpha}$ & 0.64 \\
\hline
\end{tabular}

controlled by the droop control strategy. Therefore, all DG units operate according to their droop coefficients. In this case, there is no guarantee that overloading of resources can be prevented via droop control strategy. It is up to the decision of MG operator to use Load 1 (L1), Load 2 (L2), Load 31 (first fraction of L3),

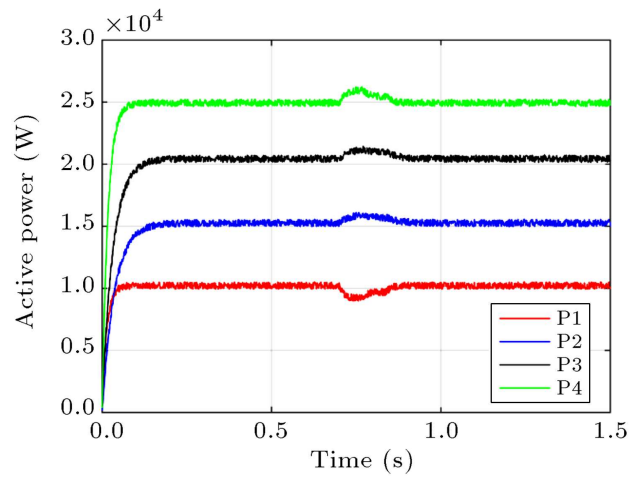

(a)

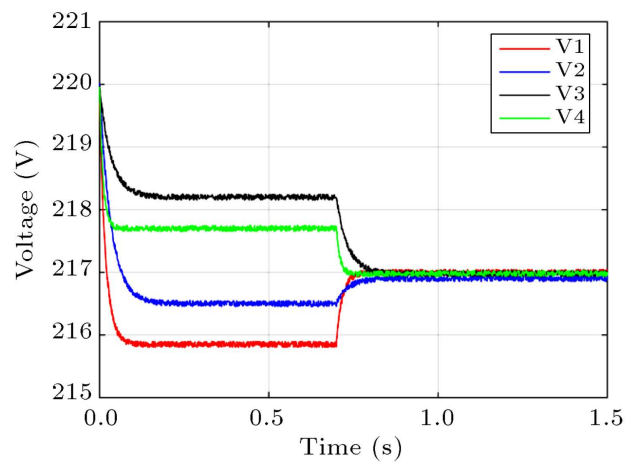

(c)
Load 32 (second fraction of L3), and Load 4 (L4) in MG. Active and reactive powers are shared among DG units, as shown in Figure 5(a) and (b). When $t=0.7 \mathrm{~s}$, the droop control strategy is switched to the proposed method. It is observed that the active powers return to the same as initial values after a transient condition. However, the reactive powers will be shared based on the DG's nominal capacity of ratio $1: 1.5: 2$ : 2.5 . Therefore, DG1, which has previously been exposed to overload, reduces its reactive power. DGs 2,3 , and 4 also provide the required powers based on their nominal capacity ratio.

Figure 5(c) also shows the voltage profile of terminals connected to DG units. In this figure, due to the inadequate share of reactive power by the droop method before $t=0.7 \mathrm{~s}$, the system voltage will not remain at a constant value. However, following the implementation of the proposed method, the reactive power helps the terminal voltages remain at a nominal value. The system frequency during this study is given in Figure 5(d), which confirms the proper performance of the proposed method. The system frequency is close to $50 \mathrm{~Hz}$ during the simulation period.

\subsection{Case study 2}

In this case, it is assumed that the system is operated by the proposed controller. According to the archived

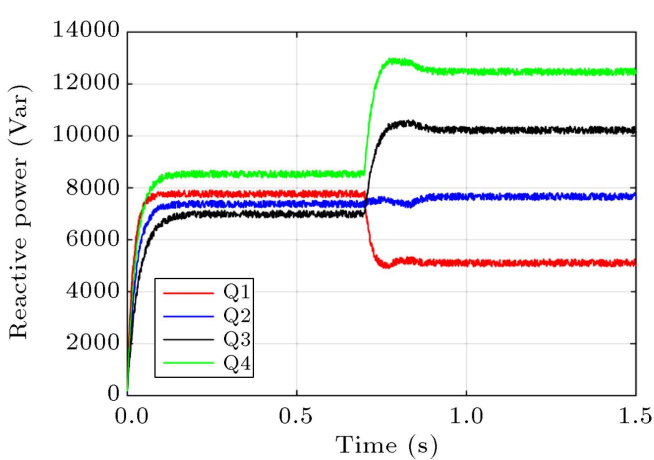

(b)

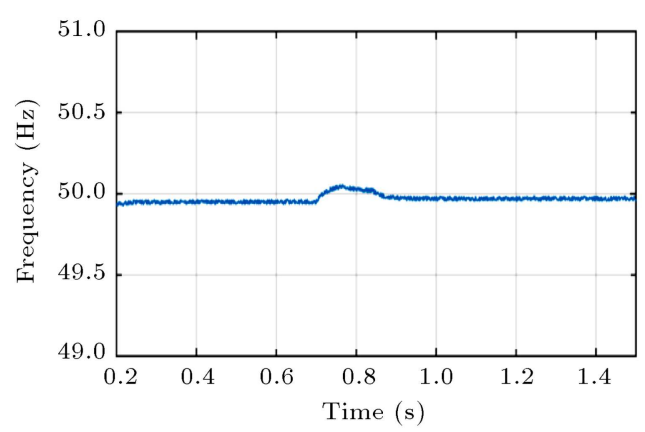

(d)

Figure 5. (a) Active power sharing, (b) reactive power sharing, (c) terminal voltage of Distributed Generation (DG) units, and (d) Micro-Grid (MG) frequency. All plots are calculated by droop method before $t=0.7 \mathrm{~s}$, and proposed method after $t=0.7 \mathrm{~s}$. 
results, the proposed controller can share active and reactive power among the DG units in an acceptable manner. At $t=0.6 \mathrm{~s}$, the local load is connected to the MG. The proposed controller shares the power among the DG units in the shortest possible amount of time based on their nominal capacity. Therefore, active and reactive powers are shared successfully and effectively among all DG units. Figure 6(a) and (b) show the output active and reactive powers of $D G$ units, respectively. As can be seen, in the case of reactive power sharing, the nominal capacity ratio of DGs is also obtained. This prevents the overloading of DG units. Figure 6(c) represents the terminal voltage profile. When the local load is connected to $\mathrm{MG}$, the voltages of MG buses are reduced slightly. When $t=1 \mathrm{~s}$, the local load and Load L32 are switched off. Therefore, the proposed controller shares the remainder of active and reactive powers among DG units properly to their nominal capacity ratio. Figure 6(c) shows an increase in voltage amplitude due to a decrease in local load. Figure 6(d) shows slight variations in $\mathrm{MG}$ frequency from its nominal value.

\subsection{Case study 3}

Similar to the first case study, the MG starts working using the droop control method. At $t=0.7 \mathrm{~s}$, the droop approach is switched off and the proposed controller

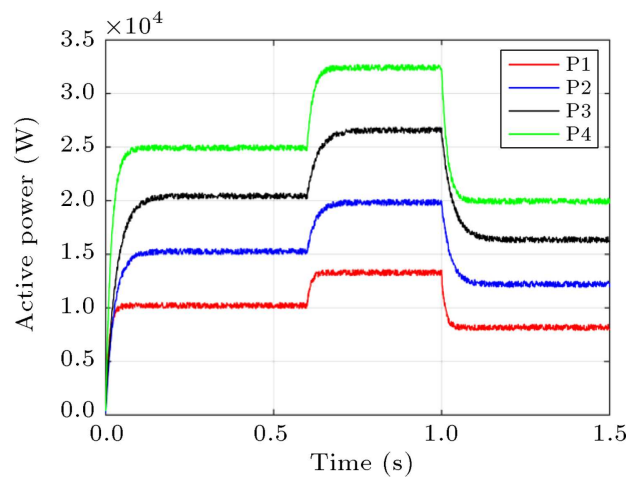

(a)

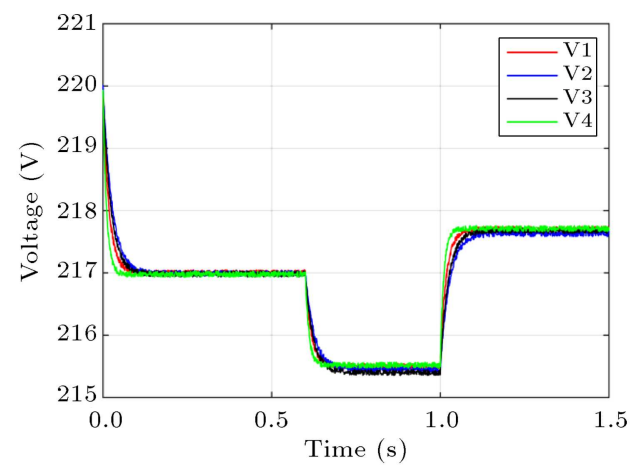

(c) is substituted. Up to this point, it is similar to the first case. At $t=1.4 \mathrm{~s}$, the protective relays of DG2 disconnect it from the network. Thus, its active and reactive powers are degraded to zero, as carefully illustrated in Figure $7(\mathrm{a})$ and (b). Afterwards, the terminal voltage of DG2 will be equal to zero and the open-circuit voltage will rise to $220 \mathrm{~V}$. Moreover, other DG units share the load power with the nominal ratio of 1: 2: 2.5. This means that the output power of each resource increases without any overloading in DG units output powers. The active and reactive powers are shown after $t=1.4 \mathrm{~s}$ in Figure $7(\mathrm{a})$ and (b), respectively. In this regard, $70 \mathrm{MW}$ load with ratios of 12.72: 25.45: 31.81 is shared among DG1, DG3, and DG4, respectively. Moreover, the remainder of reactive power is shared via DG1, DG3, and DG4 by the ratio of 6.36 : 12.72: 15.9 , which is equal to their nominal capacity ratio of $1: 2: 2.5$. Figure 7 (c) represents the terminal voltage of all DG units and $M G$ frequency is plotted in Figure $7(\mathrm{~d})$.

In order to compare the performance of the proposed controller with those of some improved SMC approaches, i.e., Adaptive Sliding Mode Control (ASMC) and Adaptive Fuzzy Sliding Mode Control (AFSMC), a worthy comparison based on the step response is made, as shown in in Figure 8 . According to this figure, the proposed strategy has the best settling time,

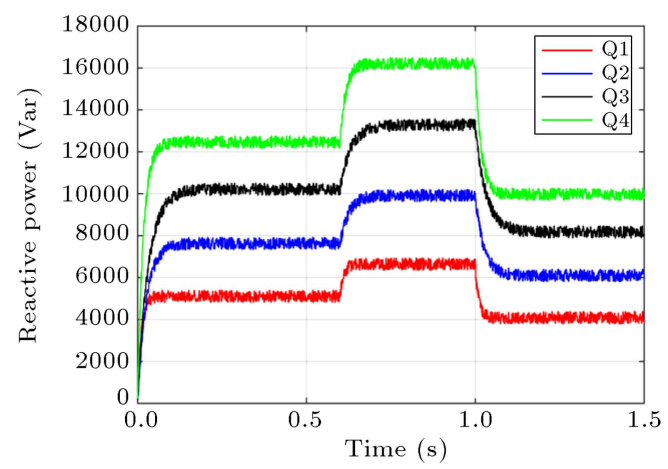

(b)

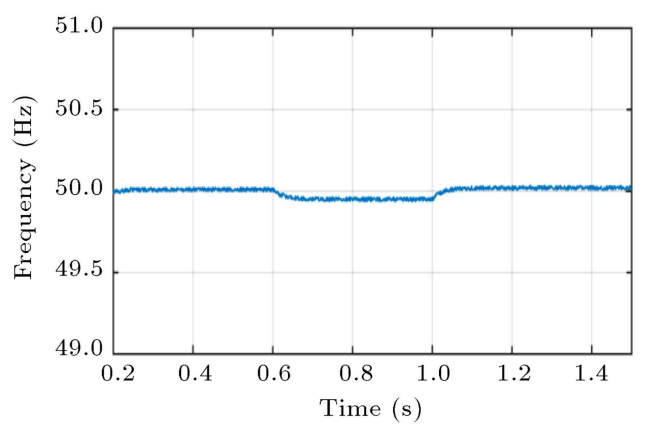

(d)

Figure 6. (a) Active power sharing, (b) reactive power sharing, (c) terminal voltage of Distributed Generations (DGs), and (d) Micro-Grid (MG) frequency. All plots are calculated by the proposed controller with contingency of load increasing at $t=0.6 \mathrm{~s}$ and decreasing at $t=1 \mathrm{~s}$. 


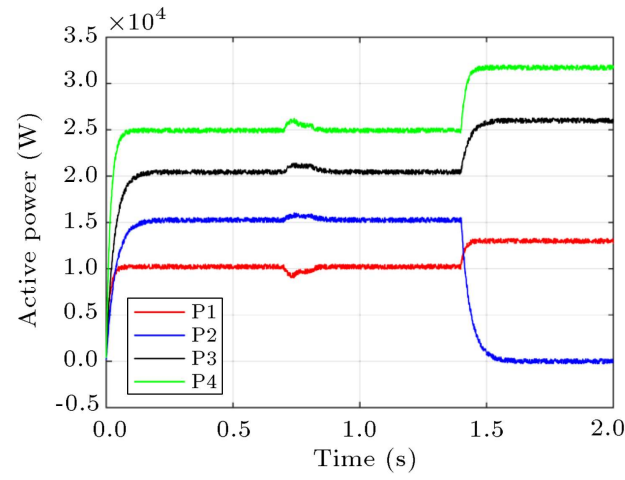

(a)

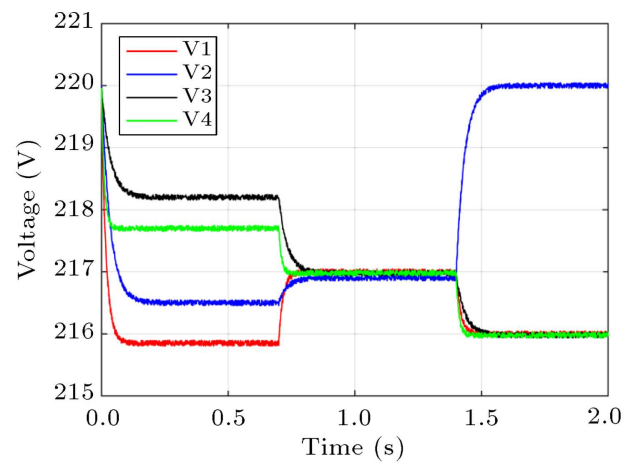

(c)

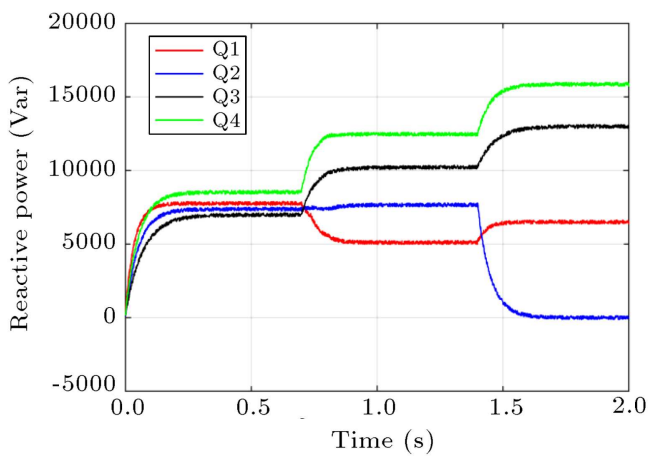

(b)

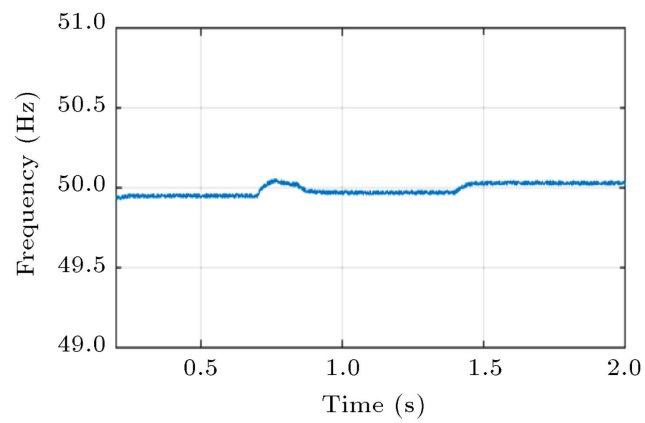

(d)

Figure 7. (a) Active power sharing, (b) reactive power sharing, (c) terminal voltage of Distributed Generations (DGs), and (d) Micro-Grid (MG) frequency. All plots are calculated by droop method before $t=0.7 \mathrm{~s}$ and the proposed method after $t=0.7 \mathrm{~s}$. Power outage occurs at $t=1.4 \mathrm{~s}$.

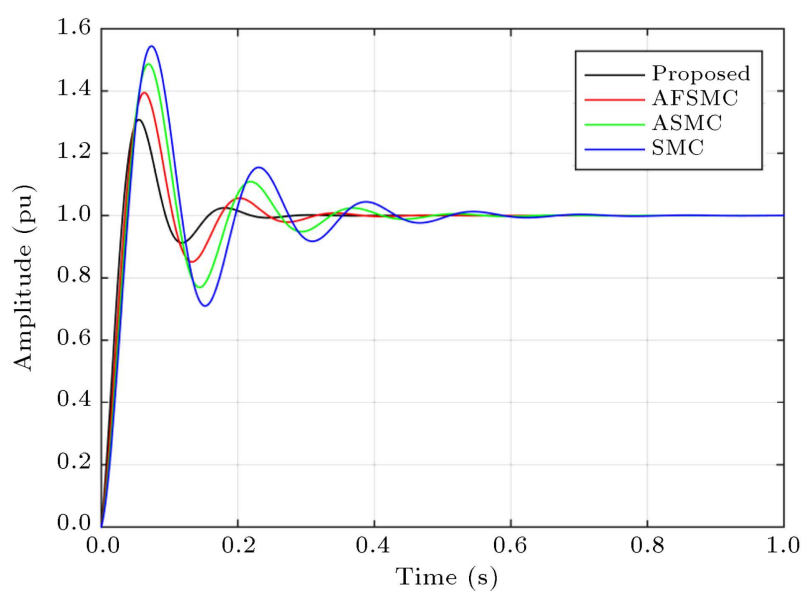

Figure 8. Step responses of the proposed controller strategy as well as Adaptive Fuzzy Sliding Mode Control (AFSMC), Adaptive Sliding Mode Control (ASMC), and Sliding Mode Control (SMC) approaches.

over-shoot, and rise time values. The settling time durations of the proposed controller, AFSMC, ASMC, and SMC approaches are $0.347 \mathrm{~s}, 0.412 \mathrm{~s}, 0.595 \mathrm{~s}$, and $0.661 \mathrm{~s}$, respectively. It is implied that the proposed controller converges to its final value more quickly than the others. By the way, shorter rise time and over-shoot indicate that our controller presents a better solution effectively.
Given that this control strategy is quite useful for dynamic stability analysis of power systems, some control indices are expressed in Eqs. (41) to (43) and all of these evaluations are summarized in Tables 7 and 8. In this part, it is assumed that a load variation between $-0.1 \mathrm{pu}$ and $+0.1 \mathrm{pu}$ occurs. Table 8 confirms that our approach has the best performance among the others.

$$
\begin{aligned}
& \text { Integral Sqaure Error } \rightarrow \mathrm{ISE}=\int_{0}^{\infty} e(t)^{2} d t \\
& \text { Integral Absolute Error } \rightarrow \mathrm{IAE}=\int_{0}^{\infty}|e(t)| d t, \\
& \text { Integral Time Absolute Error } \rightarrow \mathrm{ITAE} \\
& \quad=\int_{0}^{\infty} t|e(t)| d t .
\end{aligned}
$$

\section{Conclusion}

In this paper, a novel and improved control method based on the sliding mode scheme was presented that would allow the central controller to achieve power sharing carefully among Distributed Generation (DG) units. When disturbances occur in an islanded Micro- 
Table 7. Comparison of the proposed controller with the developed Sliding Mode Control (SMC) method.

\begin{tabular}{cccc}
\hline Step response & Over shoot & Settling time & Rise time \\
\hline SMC [11] & $1.532(\mathrm{pu})$ & $0.661(\mathrm{~s})$ & $0.878(\mathrm{~s})$ \\
ASMC [12] & $1.492(\mathrm{pu})$ & $0.595(\mathrm{~s})$ & $0.810(\mathrm{~s})$ \\
AFSMC [13] & $1.398(\mathrm{pu})$ & $0.412(\mathrm{~s})$ & $0.741(\mathrm{~s})$ \\
Proposed approach & $1.506(\mathrm{pu})$ & $0.347(\mathrm{~s})$ & $0.655(\mathrm{~s})$ \\
\hline
\end{tabular}

Table 8. Control stability indices comparison.

\begin{tabular}{ccccccccccc}
\hline & \multicolumn{4}{c}{ Proposed approach } & \multicolumn{4}{c}{ SMC [11] } & \multicolumn{3}{c}{ PI } \\
\hline$\Delta P_{L}$ & ISE & IAE & ITAE & ISE & IAE & ITAE & ISE & IAE & ITAE \\
$-0.1 \mathrm{pu}$ & 0.017 & 0.023 & 0.0087 & 0.028 & 0.034 & 0.012 & 0.044 & 0.057 & 0.019 \\
$+0.1 \mathrm{pu}$ & 0.036 & 0.033 & 0.011 & 0.048 & 0.049 & 0.025 & 0.071 & 0.082 & 0.039 \\
\hline
\end{tabular}

Grid (MG), preserving the stability of the MG is considered as the vital principle. Therefore, the proposed controller with internal loop controls the voltage and frequency of the MG within an acceptable range. Afterwards, the secondary control loop begins the power sharing among DGs. The achieved results showed that power and voltage disturbances could be easily eliminated and reduced perfectly. Thus, the MG frequency was kept constant and very close to its nominal value. The sliding mode control method can be more effective than intelligent control fashions due to its ability to damp perturbations. Finally, after making the comparisons, fuzzy or adaptive methods cannot properly handle DG units as good as the presented method. The constant inverter frequency acts as another reason for the superiority of the proposed strategy.

\section{References}

1. Akhavan, A. and Mohammadi, H.R. "A new control method for grid-connected quasi-Z-source multilevel inverter based photovoltaic system", Scientia Iranica, Transactions D, Computer Science \& Engineering, Electrical, 22(6), p. 2505 (2015).

2. Akhbari, A. and Rahimi, M. "Performance and stability analysis of grid connected single phase inverters used in solar photovoltaic systems", Scientia Iranica, Transactions D, Computer Science \& Engineering, Electrical, 26(3), pp. 1637-1651 (2017).

3. Farahani, H.F., Khalili, M., Rabiee, A., et al. "On the application of plug-in hybrid electric vehicle to compensate network harmonics: A multiobjective approach", Scientia Iranica, Transaction D, Computer Science and Engineering, Electrical, 21(6), pp. 21772185 (2014).

4. Asad, R. and Kazemi, A. "A novel practical fair nodal price for DC microgrids and distribution systems"
Scientia Iranica, Transaction D, Computer Science \& Engineering, Electrical, 21(6), pp. 2232-2242 (2014).

5. D'Arco, S. and Suul, J.A. "Equivalence of virtual synchronous machines and frequency-droops for converterbased microgrids", IEEE Transactions on Smart Grid, 5(1), pp. 394-395 (2014).

6. Fang, T., Shen, L., He, W., et al. "Distributed control and redundant technique to achieve superior reliability for fully modular input-series-output-parallel inverter system", IEEE Transactions on Power Electronics, 32(1), pp. 723-735 (2017).

7. Cingoz, F., Elrayyah, A., and Sozer, Y. "Optimized droop control parameters for effective load sharing and voltage regulation in DC microgrids", Electric Power Components and Systems, 43(8-10), pp. 879889 (2015).

8. de Souza, W.F., Severo-Mendes, M.A., and Lopes, L.A. "Power sharing control strategies for a threephase microgrid in different operating condition with droop control and damping factor investigation", IET Renewable Power Generation, 9(7), pp. 831-839 (2015).

9. Nasirian, V., Shafiee, Q., Guerrero, J.M., et al. "Droop-free distributed control for AC microgrids", IEEE Transactions on Power Electronics, 31(2), pp. 1600-1617 (2016).

10. Palizban, O. and Kauhaniemi, K. "Hierarchical control structure in microgrids with distributed generation: Island and grid-connected mode", Renewable and Sustainable Energy Reviews, 44(1), pp. 797-813 (2015).

11. Milczarek, A., Malinowski, M., and Guerrero, J.M. "Reactive power management in islanded microgridProportional power sharing in hierarchical droop control", IEEE Transactions on Smart Grid, 6(4), pp. 1631-1638 (2015).

12. Gudey, S.K. and Gupta, R. "Recursive fast terminal sliding mode control in voltage source inverter for 
a low-voltage microgrid system", IET Generation, Transmission and Distribution, 10(7), pp. 1536-1543 (2016).

13. Huang, Y.J., Kuo, T.C., and Chang, S.H. "Adaptive sliding-mode control for nonlinearsystems with uncertain parameters", IEEE Transactions on Systems, Man, and Cybernetics, Part B (Cybernetics), 38(2), pp. 534-539 (2008).

14. Ghiasi, M.I., Golkar, M.A., and Hajizadeh, A. "Lyapunov based-distributed fuzzy-sliding mode control for building integrated-DC microgrid with plug-in electric vehicle", Ieee Access, 5, pp. 7746-7752 (2017).

15. He, J., Li, Y.W., and Blaabjerg, F. "An enhanced islanding microgrid reactive power, imbalance power, and harmonic power sharing scheme", IEEE Transactions on Power Electronics, 30(6), pp. 3389-3401 (2015).

16. Zhong, Q.C., Wang, Y., and Ren, B. "UDE-based robust droop control of inverters in parallel operation", IEEE Transactions on Industrial Electronics, 64(9), pp. 7552-7562 (2017).

17. Han, H., Liu, Y., Sun, Y., et al. "An improved droop control strategy for reactive power sharing in islanded microgrid", IEEE Transactions on Power Electronics, 30(6), pp. 3133-3141 (2015).

18. Mahmood, H., Michaelson, D., and Jiang, J. "Accurate reactive power sharing in an islanded microgrid using adaptive virtual impedances", IEEE Transactions on Power Electronics, 30(3), pp. 1605-1617 (2015).

19. Guerrero, J.M., Loh, P.C., Lee, T.L., et al. "Advanced control architectures for intelligent microgrids-Part II: Power quality, energy storage, and AC/DC microgrids", IEEE Transactions on Industrial Electronics, 60(4), pp. 1263-1270 (2013).

20. Krishnasamy, V.S., Mashayekhi, S., and Razzaghi, M. "Numerical solutions of fractional differential equations by using fractional Taylor basis", IEEE/CAA Journal of Automatica Sinica, 4(1), pp. 98-106 (2017).

21. Bekir, A., Guner, O., and Cevikel, A. "The expfunction method for some time-fractional differential equations", IEEE/CAA Journal of Automatica Sinica, 4(2), pp. 315-321 (2017).

22. Salehi, Y. and Schiesser, W.E. "Numerical integration of space fractional partial differential equations: vol 2-applications from classical integer PDEs", Synthesis Lectures on Mathematics and Statistics, 9(3), pp. 1205 (2017).

23. Patnaik, R.K. and Dash, P.K. "Fast adaptive backstepping terminal sliding mode power control for both the rotor-side as well as grid-side converter of the doubly fed induction generator-based wind farms", IET Renewable Power Generation, 10(5), pp. 598-610 (2016).

24. Mobayen, S. "An adaptive chattering-free PID sliding mode control based on dynamic sliding manifolds for a class of uncertain nonlinear systems", Nonlinear Dynamics, 82(1-2), pp. 53-60 (2015).

25. Gallegos, J.A. and Duarte-Mermoud, M.A. "On the Lyapunov theory for fractional order systems", $A p$ plied Mathematics and Computation, 287, pp. 161-170 (2016).

\section{Biographies}

Mohammad Amin Bina was born in 1985 in Iran. He received his BSc degree in Electrical Engineering from Islamic Azad University of Kazeroun and the MS degree from Islamic Azad University of Sari in 2008 and 2013, respectively. Currently, he is working at Electricity Distribution Company of Fars Province for more than 10 years. His research interests are distribution systems maintenance, $\mathrm{AC} / \mathrm{DC} / \mathrm{AC}$ converters, and photovoltaic system control and operation.

Mohsen Gitizadeh was born in Iran in 1976. He received his BS degree in Electrical Engineering from Shiraz University in 1999 and his MS and PhD degrees from Iran University of Science and Technology in 2001 and 2009, respectively. Dr. Gitizadeh is currently an Associated Professor at the Department of Electrical and Electronics Engineering at Shiraz University of Technology in Shiraz, Iran. His current research interests are in the areas of power system operation and control, energy conversion, voltage stability and optimization, demand side management, and FACTS devices.

Mehrdad Mahmoudian was born in Iran in 1990. $\mathrm{He}$ received the $\mathrm{BSc}$ degree in Electrical Engineering from Shahid Bahonar University of Kerman and MS degree in Electrical Engineering from Iran University of Science and Technology (IUST) in 2012 and 2014, respectively. Currently, he is working towards the PhD degree in Electrical Engineering at Shiraz University of Technology, Shiraz, Iran. His research interests include $\mathrm{DC} / \mathrm{DC}$ converters, DC/AC inverters, energy conversion, and photovoltaic power system. 